\title{
25 Research Square \\ Integration of Transcriptome and Proteome Analysis \\ Reveals the Mechanism of Freezing Tolerance in Winter \\ Rapeseed
}

\section{Jiaping Wei}

Gansu Agricultural University

\section{Guoqiang Zheng}

Gansu Agricultural University

\section{Xiaoyun Dong}

Gansu Agricultural University

Hui Li

Gansu Agricultural University

\section{Sushuang Liu}

Gansu Agricultural University

\section{Ying Wang}

Gansu Agricultural University

\section{Zigang Liu ( $\sim$ lzgworking@163.com )}

Gansu Agricultural University https://orcid.org/0000-0001-7271-393X

\section{Research Article}

Keywords: winter rapeseed, freezing stress, transcriptome, proteome, physiology, ultrastructure

Posted Date: August 26th, 2021

DOI: https://doi.org/10.21203/rs.3.rs-752901/v1

License: @ (i) This work is licensed under a Creative Commons Attribution 4.0 International License. Read Full License

Version of Record: A version of this preprint was published at Plant Growth Regulation on October 25th, 2021. See the published version at https://doi.org/10.1007/s10725-021-00763-z. 


\section{Abstract}

Winter rapeseed seedlings are susceptible to low temperature during overwintering in Northwest China, leading to reduced crops production. Freezing stress is one of the utmost environmental stresses in Northwest China from late autumn to early spring, which are eventful for overwinter survival rate of winter rapeseed. However, the molecular mechanism of freezing tolerance formation is still very backward in winter rapeseed. In this study, using a pair of freezing-sensitive and freezing-resistant cultivars NQF24 and NTS57, the exhaustive effects of freezing stress on freezing tolerance formation were evaluated by analyzing leaf at the levels of transcriptome, proteome, physiology and ultrastructure. There were 8497 differentially expressed genes (DEGs) and 418 differentially abundant proteins (DAPs) specifically identified in leaf of NQF24 under freezing stress, while 7358 DEGs and 573 DAPs were specifically identified in leaf of NTS57. Function enrichment analysis showed that most of the enriched DEGs and DAPs were associated with plant hormones signal transduction, fatty acid metabolism, ribosome, plant-pathogen interaction and secondary metabolites biosynthesis. Freezing tolerance is formed by enhanced signals transduction, increased the biosynthesis of protein and secondary metabolites, higher reactive oxygen species (ROS) scavenging, more osmolytes, lower lipid peroxidation, and stronger ultrastructure. These results can be taken as selection indicators in freezing tolerance breeding program in rapeseed.

\section{Introduction}

Freezing stress $\left(<0^{\circ} \mathrm{C}\right)$ has profound and diverse effects on plant growth and development, it poses a serious threat to the geographical distribution of plant species (Ding et al. 2019a), it can also cause ice formation in the apoplast of plant tissues, the accumulation of intracellular ice physically disrupts the cell membrane (Shi et al. 2018), leading to severe cell dehydration. In the area of Northwest China, which could not only impact on the quality of crops, but also reduce the production of crops, especially for winter crops grown in high latitudes and altitudes regions (Xu et al. 2018). Some previous studies in model plants have shown that the adaptation of the plant to freezing stress must change gene expression at the cellular level, which in turn alters the protein profiles (Ding et al. 2015; Zhang et al. 2017). However, to date, the limited information is available about freezing-response genes or proteins in winter rapeseed.

Winter rapeseed (Brassica napus L.) is an important oilseed crop that is widely cultivated in Northwest China. However, its growth is easily persecuted to low temperature in Northwest China, which prevents winter rapeseed from overwinter and propagate, leading to severe reduction of crops yield. As a result, it is critical to acquire strong freezing resistance for winter rapeseed so as to overwinter safely. In recent years, the studies by Xu et al. (2018) and Zeng et al. (2018) suggested the impacts on the roots and leaves of two winter rapeseed cultivars after freezing treatment by isobaric tags for relative and absolute quantification. Pu et al. (2019) reported the effects on the freezing resistance formation in the leaves of two rapeseed cultivars after freezing treatment by high-throughput RNA sequencing (RNA-seq). However, all the above researches are based on traditional data-dependent acquisition (DDA) models, which might result in lower accuracy in the quantification of low abundance proteins. The data-independent acquisition (DIA) technique has an excellent detection rate, and it is considered as one of the most striking techniques by the researchers in Nature Methods (Egertson et al. 2013). Our recent study obtained a freezing-responsive molecular network after short-term successive freezing treatment by the sequencing-based transcriptome and DIA-based proteome (Wei et al. 2021).

In the present study, under long-term repeated freezing stress, on the one hand, we analyzed the physiological, biochemical and ultrastructural properties in the leaves of resistant and susceptible winter rapeseed cultivars; on the other hand, based on the results of DNA methylation analysis, the combined analysis of transcriptomics and proteomics was used to identify the freezing-responsive DEGs and DAPs in the leaves of two winter rapeseed cultivars; thereafter, the main metabolic pathways, cellular processes and differentially expressed transcription factors (TFs) associated with freezing stress were investigated; finally, we got a sophisticated molecular regulatory network of winter rapeseed in response to long-term freezing stress, it will be helpful to expedite the breeding and production of winter rapeseed cultivars with strong freezing tolerance. 


\section{Materials And Methods \\ Plant samples and freezing stress treatments}

A pair of winter rapeseed cultivars freezing-resistant NTS57 (NS) and freezing-sensitive NQF24 (NF) used in the present study, were produced by Gansu Agricultural University (Wei et al. 2021). Seedlings of the two cultivars were grown in $5 \mathrm{~L}$ plastic pots for 5 weeks under constant $22^{\circ} \mathrm{C}$ and $16 \mathrm{~h} / 8 \mathrm{~h}$ (day/night) cycle with $350 \mu \mathrm{mol} \cdot \mathrm{m}^{-2} \cdot \mathrm{s}^{-1}$ light. Subsequently, potted plants were divided into two groups for freezing treatments. Briefly, the plants in freezing treatment group (Treatment, T) were transferred to an artificial climate room $\left[-4^{\circ} \mathrm{C} / 22{ }^{\circ} \mathrm{C}, 12 \mathrm{~h} / 12 \mathrm{~h}\right.$ (day/night) cycle with $350 \mu \mathrm{mol} \cdot \mathrm{m}^{-}$ $2 . \mathrm{s}^{-1}$ light] for 2 day. The plants in control group (Control, $\mathrm{C}$ ) at the equal growth stage were placed in a separate chamber at $22{ }^{\circ} \mathrm{C}$ for 2 day $\left[12 \mathrm{~h} / 12 \mathrm{~h}\right.$ (day/night) cycle with $350 \mu \mathrm{mol} \cdot \mathrm{m}^{-2} \cdot \mathrm{s}^{-1}$ light]. The third leaf was selected after treatment with three biological replicates. Fresh samples were used for determination of net photosynthetic rate and transmission electron microscope (TEM) observations, while another set of samples were refrigerated in liquid nitrogen instantly and preserved at $-80^{\circ} \mathrm{C}$ for total RNA and proteins extraction, and physiological and biochemical measurements.

\section{Determination of net photosynthetic rate}

Net photosynthetic rate was measured according to Ma et al. (2017) with some modifications. Photosynthesis was evaluated using a LI-COR 6400 portable gas analysis system (Lincoln, NE, USA) setting a built-in light source of 1000 $\mu \mathrm{mol}$ photons $\mathrm{m}^{-2} \cdot \mathrm{s}^{-1}$. Each sample was repeatedly determined six times during 10:00 am and 11:00 am to minify the error.

\section{TEM observations}

Fresh rapeseed leaves from treatments and controls were used for TEM observations. Each sample was cut into small size of $2 \mathrm{~mm}^{2}$ in the $2.5 \%$ glutaraldehyde fixative for $16 \mathrm{~h}$ at $4^{\circ} \mathrm{C}$, followed by a post-fixation with $1 \% \mathrm{OsO}_{4}$ for $2 \mathrm{~h}$ at room temperature. All fixed samples were dehydrated in a graded series of ethanol and embedded in Spurr resin. The thin ultrathin Sect. 60-80 nm were finally stained with $2 \%$ uranium acetate saturated alcohol solution avoid light for 10 min and $2.6 \%$ lead citrate avoid $\mathrm{CO}_{2}$ for $10 \mathrm{~min}$, and then rinsed with ultra-pure water for 3 times. Observations were manipulated on an H-7650 electron microscope. High-resolution TEM images were produced as described in Toyooka et al. (2014).

\section{Physiological and biochemical measurements}

Rapeseed leaves $(0.1 \mathrm{~g})$ were used for measuring physiological and biochemical properties, including antioxidant enzymes [catalase (CAT), peroxidase (POD) and superoxide dismutase (SOD)] activities, lipid peroxidation level, and the contents of osmotic regulation substances (soluble sugar and protein). The antioxidant enzymes activities were measured using the kits from Beijing Solarbio Science \& Technology following the manufacturer's protocols (Chen et al. 2019). The level of lipid peroxidation was evaluated by determining MDA content according to Wei et al. (2020). The contents of soluble sugar and soluble protein were measured as described by Song et al. (2015).

\section{RNA extraction and RNA sequencing in rapeseed}

The frozen leaf samples were ground in liquid nitrogen and total RNA were extracted using the TRIzol Reagent (Tiangen Biotech, China) according to the manufacturer's instructions. The sequencing library was constructed and sequenced in Gene Denovo Biotechnology Co. (Guangzhou, China) on an Illumina HiSeqTM 2500 platform. The transcriptomic raw data have been uploaded to the SRA database of NCBI (PRJNA685002).

\section{Filtering of reads and gene expression analysis}


Raw data were filtered to remove adapters, poly- $\mathrm{N}$ and low-quality reads to obtain clean reads. The high-quality paired-end reads were mapped to rapeseed reference genome (Brassica napus v2.0) using TopHat v2.0.3.12 (Kim et al. 2013). The levels of gene expression were quantified and normalized as fragments per kilobase per million mapped reads (FPKM), which can be diametrically used to identify DEGs in pair-wise comparisons (Trapnell et al. 2010). Genes with p-value < 0.001 and value of $\mid \log _{2}$ FoldChange| $\geq 2$ were regarded as DEGs using the edgeR package (http://www.r-project.org/).

\section{Protein extraction and digestion}

Total protein was extracted using the chilled acetone precipitation method according to Wei et al. (2020) with some changes. Briefly, samples were firstly ground into powder in liquid nitrogen, then dissolved in $2 \mathrm{~mL}$ lysis buffer containing 8 M Urea, 2\% SDS, $1 \times$ Protease Inhibitor Cocktail, followed by sonication on ice for 20 min and centrifugation at 12000 rpm for $10 \mathrm{~min}$ at $4{ }^{\circ} \mathrm{C}$. The supernatant was pipetted into a new centrifuge tube. Protein was precipitated with chilled acetone for $16 \mathrm{~h}$ at $-20^{\circ} \mathrm{C}$, cleaned with acetone two times, re-dissolved in $8 \mathrm{M}$ Urea, and homogenized in ice for $3 \mathrm{~min}$ using an ultrasonic homogenizer. The homogenate was centrifuged at $12000 \mathrm{rpm}$ for $15 \mathrm{~min}$ at $4{ }^{\circ} \mathrm{C}$, then the supernatant was preserved and the protein concentration was gauged by the BCA Protein Assay Kit (Thermo Fisher, Waltham, MA). A total of $50 \mu \mathrm{g}$ protein were suspended in $50 \mu \mathrm{L} 50 \mathrm{mM}$ ammonium bicarbonate, reduced by $10 \mathrm{mM}$ dithiothreitol for $1 \mathrm{~h}$ at $55^{\circ} \mathrm{C}$ and alkylated by $20 \mathrm{mM}$ iodoacetamide for $1 \mathrm{~h}$ at $37^{\circ} \mathrm{C}$ in the dark. Thereafter, each sample was precipitated with $500 \mathrm{ul}$ chilled acetone for $16 \mathrm{~h}$ at $-20^{\circ} \mathrm{C}$. The precipitates were washed three times with chilled acetone and resuspended in $50 \mathrm{mM}$ ammonium bicarbonate. Finally, all samples were digested with sequencing-grade modified trypsin (Promega, Madison, $\mathrm{WI}$ ) at a substrate/enzyme ratio of $50: 1$ (w/w) at $37^{\circ} \mathrm{C}$ for $16 \mathrm{~h}$.

\section{High PH reverse phase separation and LC-MS/MS analysis}

Digested peptides from each sample were segregated on an Ultimate 3000 system (Thermo Fisher, USA) with a high pH reversed phase XBridge BEH C18 column $(4.6 \mathrm{~mm} \times 250 \mathrm{~mm}, 5 \mu \mathrm{m})$. High pH separation was performed using a linear gradient, starting from $5 \% \mathrm{~B}(20 \mathrm{mM}$ ammonium formate in $80 \%$ acetonitrile, $\mathrm{pH} 10.0)$ to $45 \% \mathrm{~B}$ in 40 min. Peptides were eluted at a flow rate of $1 \mathrm{~mL} / \mathrm{min}$. For each sample, 10 fractions were convened and dried by a vacuum pump. All dried samples were saved at $-80{ }^{\circ} \mathrm{C}$ until the next analysis.

The dried peptides were dissolved in $30 \mu \mathrm{L}$ buffer $\mathrm{D}(0.1 \%$ formic acid and $0.1 \%$ formic acid in acetonitrile). LC-MS/MS analysis was operated on an Orbitrap Fusion Mass Spectrometer (Thermo Fisher, Germany) coordinated with a Nano ACQUITY UPLC system (Waters, Milford, MA). In short, $10 \mu \mathrm{L}$ peptides were added to the trapping column (Acclaim PepMap C18, $100 \mu \mathrm{m} \times 2 \mathrm{~cm}$ ) and then eluted on an analytical column (Acclaim PepMap C18, $75 \mu \mathrm{m} \times 25 \mathrm{~cm}$ ) by a 120 minutes gradient of buffer $\mathrm{D}$. The flow rate of column was retained at $500 \mathrm{~nL} / \mathrm{min}$, and the electrospray voltage was set to $2.1 \mathrm{kV}$.

For library generation, to acquire data in DDA mode, the Orbitrap Lumos was performed in a positive mode. MS spectra $(350-1500 \mathrm{~m} / \mathrm{z})$ were collected at $1.2 \times 10^{5}$ resolution to attain an automatic gain control (AGC) target of $4.0 \times 10^{5}$. The precursor ions were used for MS/MS detection with a collision energy of 32. Dynamic exclusion was set to $30 \mathrm{~s}$ to exclude all isotopes. MS/MS spectra were collected at $1.5 \times 10^{4}$ resolution to attain an AGC target of $5.0 \times 10^{4}$. For data acquisition, we used DIA mode. MS spectra (350-1500 m/z) were collected at $1.2 \times 10^{5}$ resolution to attain an AGC target of $5.0 \times 10^{5}$. MS/MS spectra were collected at $3.0 \times 10^{4}$ resolution to attain an AGC target of $1.0 \times 10^{6}$ with a collision energy of 32. All 60 segments were selected for MS/MS. The acquisition window covered $1 \mathrm{~m} / \mathrm{z}$ through 60 consecutive isolation windows.

\section{Mass spectra data and protein quantification}

Spectronaut Pulsar 11.0 software (Bruderer et al. 2015) was applied to analyze DDA MS/MS data, and the settings were as follows: digestion enzyme, trypsin; fixed modification, carbamidomethylation (C); variable modifications, oxidation (M); precursor mass tolerance, 20 ppm; fragment mass tolerance, 0.05 Da. The MS/MS data were searched from Brassica 
napus database (Brassica napus v2.0; Table S1), which were downloaded from the NCBI on May, 2018 with 123465 sequences. False discovery rates of all peptides were set to $1 \%$, which were evaluated by a reverse search sequence. Subsequently, raw data form each sample with the spectra library based on DIA MS/MS data were analyzed by Spectronaut Pulsar 11.0 with default parameters depend on the previously constructed DDA protein dataset. All proteins quantification normalization were performed by the local normalization with Pulsar software. The MS raw data have been submitted into a public iProX database (Ma et al. 2019a) with the accession number of IPX0002682000. Proteins with pvalue $<0.001$, peptides $\geq 2$ and value of $\mid \log _{2}$ FoldChangel $\geq 1$ were regarded as DAPs using the edgeR package.

\section{Bioinformatics and statistics analysis}

The correlation coefficient between three replicates was analyzed to assess the repeatability of the experimental results. Principal component analysis (PCA) was carried out to disclose the relationship between samples by R package (http://www.r-project.org/). Genes and proteins were annotated using the Gene Ontology (GO) database (http://geneontology.org/) and the Kyoto Encyclopedia of Genes and Genomes (KEGG) database (http://www.kegg.jp/) to obtain their functions (Binns et al. 2009; Kanehisa et al. 2016). Enrichment analysis of DEGs and DAPs were conducted on the basis of GO database and KEGG database. All physiological data were performed one-way analysis of variance and mean differences of three biological replicates were obtained by LSD test.

\section{Results}

\section{Physiological and biochemical responses to freezing stress}

NF plants exhibited more badly languish than NS plants after freezing treatment (Fig. 1A). Compared to the controls, freezing stress provoked a significant reduction in the net photosynthetic rate of both rapeseed cultivars, but the net photosynthetic rate of rapeseed cultivar NF was significantly $(p<0.05)$ lower than that of cultivar NS under freezing treatment (Fig. 1B). In addition, TEM micrographs analysis was conducted in both cultivars after freezing treatment. Compared to the controls, the chloroplasts in mesophyll cells were brutally collapsed in cultivar NF under freezing treatment, leading to thylakoid lamellaes exposing, whereas, more plastoglobuli were observed in cultivar NS under freezing treatment (Fig. 1C). These results suggested that the freezing treatment resulted in a great injury to the leaves of NF than that of NS.

The enzyme activities of CAT and SOD in the leaf of both cultivars were found to be significantly decreased after freezing treatment, but the CAT activity in cultivar NS was significantly $(p<0.05)$ higher than that of cultivar (Fig. 2AB). The POD activity and MDA content in the leaf of both cultivars were significantly $(p<0.05)$ increased after freezing treatment, but the content of MDA in cultivar NF was significantly $(p<0.05)$ higher than that of cultivar NS (Fig. 2CD), indicating that the freezing stress resulted in more serious lipid peroxidation in cultivar NF plants. Furthermore, no obvious changes in soluble sugar content were found in both cultivars, the content of protein was significantly $(p<0.05)$ increased in cultivar NS after freezing treatment (Fig. 2EF). These results indicated that higher ROS scavenging activities and more osmotic regulation substances accumulation, and lower lipid peroxidation might create stronger freezing resistance in cultivar NS under freezing stress.

\section{Quality control analysis of transcriptome and proteome in winter rapeseed}

In the current research, $80.0 \%$ transcripts were detected in the proteins (Fig. 3A). The transcriptome in each sample was determined by RNA-Seq on the Illumina platform, and expression levels were estimated by FPKM, a total of 83846 genes including 77046 known genes and 6800 new genes were detected in all samples of developing leaves of both cultivars (Fig. 3A; Table S1). A total of 29798 unique peptides from 37247 spectra, corresponding to 13933 proteins, encoded by 11147 genes in transcriptome, were identified in all samples of developing leaves of both cultivars (Fig. 3A; Table S1). 
More down-regulated DEGs and more accumulated DAPs were discovered in both cultivars (Fig. 3B; Table S3; Table S4). PCA results denoted that three biological replicates of NFC, NFT, NSC and NST had a good consistency (Fig. 3CD), and all replicates exhibited a extremely high overlap in proteome and transcriptome for each sample (Figure S1). Moreover, the consistencies between three biological replicates of NFC, NFT, NSC and NST samples were analyzed by evaluating their correlation coefficients, all of which were more than 0.9 (Figure S2; Figure S3). These results ensured the reliability and accuracy of the proteome and transcriptome analysis.

\section{Quantitative transcriptome and proteome analysis of leaves in both rapeseed cultivars under freezing treatment}

To identify DEGs and DAPs that were induced by freezing treatment, the changes of transcription level and protein relative abundance in leaves of both cultivars were analyzed by calculating the relative level ratios of treatment to control. Compared to the corresponding controls, a total of 18117 (up, 7658; down, 10459) genes and 16978 (up, 6453; down, 10525) genes were identified to be differentially expressed in leaves of cultivars NF and NS under freezing stress, respectively (Fig. 4A; Table S2). Compared to the corresponding controls, a total of 587 and 510 proteins were found to be accumulated in abundance in leaves of cultivars NF and NS under freezing stress, respectively, whereas 168 and 400 proteins were reduced in abundance in leaves of both cultivars under freezing stress (Fig. 4B; Table S3). Both DEGs and DAPs numbers were significantly varied between both cultivars, suggesting that the dynamics of gene or protein expression regulation in both cultivars were very different.

A total of 9620 shared DEGs and 337 shared DAPs were owned in NF and NS, among the shared DEGs, 4411 were upregulated and 5209 were down-regulated in NF, while 4443 were up-regulated and 5177 were down-regulated in NS (Fig. 4C; Table S4); among the shared DAPs, 288 were accumulated in abundance and 49 were reduced in NF, while 265 were accumulated in abundance and 72 were reduced in NS (Fig. 4D; Table S4); Furthermore, 8497 DEGs and 418 DAPs were specifically identified only in NFC_NFT, while 7358 DEGs and 573 DAPs were specifically identified only in NSC_NST (Fig. 4AB; Table S5), which are the molecular basis for the difference in freezing tolerance between both rapeseed cultivars. These unique DEGs and DAPs were considered as candidate genes and proteins associated with the freezing resistance of winter rapeseed.

\section{GO and KEGG analysis of DEGs and DAPs associated with freezing tolerance}

All unique DEGs and DAPs in both cultivars under freezing stress were subjected to GO classification annotation (Figure S4; Figure S5; Table S5) and KEGG enrichment analysis. Annotated DEGs and DAPs were divided into three major functional categories including biological process, cellular component and molecular function. In the cultivar NF, most DEGs and DAPs are enriched in the cellular process, metabolic process, single-organism process, response to stimulus and biological regulation in the biological process. The enrichment related to cellular component was also obtained for DEGs and DAPs, such as the cell, cell part, and organelle. The binding and catalytic activity were the largest groups in the molecular function enriched by both DEGs and DAPs. Furthermore, top 20 KEGG enrichment pathways showed that ribosome (ko03010), linoleic acid metabolism (ko00591), propanoate metabolism (ko00640), fatty acid biosynthesis (ko00061), alpha-linolenic acid metabolism (ko00592), fatty acid elongation (ko00062), and biosynthesis of secondary metabolites (ko01110) pathways were significantly ( $q<0.05)$ enriched by DEGs (Fig. 5A; Table S5), while endocytosis (ko04144), tryptophan metabolism (ko00380), fatty acid degradation (ko00071), riboflavin metabolism (ko00740), and glycine, serine and threonine metabolism (ko00260) pathways were significantly $(q<0.05)$ enriched by DAPs (Fig. 5B; Table S5).

In the cultivar NS, the similar patterns of GO enrichment resulting from the most DEGs and DAPs were found (Figure S5; Table S5). Furthermore, top 20 KEGG enrichment pathways showed that photosynthesis (ko00195), stilbenoid, 
diarylheptanoid and gingerol biosynthesis (ko00945), limonene and pinene degradation (ko00903), biosynthesis of secondary metabolites (ko01110), pentose phosphate pathway (ko00030), plant-pathogen interaction (ko04626), and circadian rhythm (ko04712) were significantly ( $\mathrm{q}$ 0.05) enriched by DEGs (Fig. 6A; Table S5), while photosynthesisantenna proteins (ko00196), lysine degradation (ko00310), glycine, serine and threonine metabolism (ko00260), and ribosome (ko03010) pathways were significantly $(q<0.05)$ enriched by DAPs (Fig. 6B; Table S5).

\section{Identification of differentially expressed TFs in leaves of both rapeseed cultivars under freezing treatment}

A total of 1518 and 1388 differentially expressed TFs were identified in transcriptome of NF and NS after freezing treatment, respectively (Table S6). In both rapeseed cultivars, most of the up-regulated TFs belonged to ethylene response factor (AP2/ERF), WRKY transcription factor (WRKY), NAC domain-containing protein (NAC), heat stress transcription factor (HSF), and protein TIFY (TIFY) families, while most of the down-regulated TFs belonged to bHLH (bHLH), zinc finger protein (C2C2), basic leucine zipper/transcription factor TGA-like (bZIP), auxin response factor/growth-regulating factor (ARF/GFP), and transcription termination factor (mTERF) families (Fig. 7AB). However, only FHA, CSD and PBF families were identified and reduced in abundance in leaves of both cultivars under freezing treatment. Our results indicated that these TFs genes are important in response to freezing stress.

\section{Discussion}

It is acknowledged that freezing stress causes serious injuries to the plant, which is general in many winter crop growing areas around China (Zhu, 2016), especially for winter rapeseed grown in Northern China (Xu et al. 2018). Some studies have suggested the effects of freezing stress on winter rapeseed resistance formation by the transcriptome, proteome and physiology (Zeng et al. 2018; Pu et al. 2019). However, the molecular mechanism and the formation of freezing tolerance in winter rapeseed are still poorly understood. The winter rapeseed cultivar NS can survive normally in winter in most northern areas from 38 degrees north latitude to 42 degrees north latitude, and its tolerance at low temperature environments is better than other types of winter rapeseed, which contains abundant cold-resistant genes. We therefore this study focus on levels of transcript, protein, and physiology and biochemistry to elucidate the molecular basis of freezing tolerance in leaves of winter rapeseed freezing-tolerant NS and sensitive NF. It will be greatly helpful to plant molecular breeding under freezing stress.

\section{Physiological and biochemical changes between both winter rapeseed cultivars after freezing treatment}

Cold stress, including chilling $\left(0-15^{\circ} \mathrm{C}\right)$ and freezing $\left(<0^{\circ} \mathrm{C}\right)$, is a substantial abiotic stress that affects the growth and agricultural productivity of plant (Guo et al. 2018). Chilling stress inhibits the membrane to fluidity, destabilizes protein complexes, and impairs photosynthesis (Ma et al. 2018), whereas freezing stress causes more serious injuries to the plant. Freezing temperature triggers intracellular ice formation, which disrupts the cell membrane, causing a decrease in the water potential outside the cell, thus leading to severe cell dehydration, and may even create cell death (Shi et al. 2018; Ding et al. 2019a). Over the past decades, it has been reported that ROS plays an important role in response to various abiotic stresses (Baxter et al. 2014). Under adverse environmental stresses, the ROS scavenging enzymes are necessary to maintain normal cellular redox homeostasis (Ray et al. 2012). In this study, POD enzyme activity was significantly increased in both rapeseed cultivars under freezing stress, but the level of enzyme activity in cultivar NS was higher than that in cultivar NF (Fig. 2C). These results were broadly in line with Zeng et al. (2018) and Ma et al. (2019b). Some previous researches reported that cold-stressed arabidopsis roots showed the low electrolyte leakage and bad cell membrane integrity (Deng et al. 2015), the rapeseed leaves exhibited more accumulation of MDA and soluble protein under freezing stress (Xu et al. 2018). In the present study, freezing stress induced the production of MDA, soluble sugar 
and soluble protein in leaves of both cultivars, the level of lipid peroxidation in cultivar NS was significantly lower than that in cultivar NF (Fig. 2D), while the contents of soluble sugar and soluble protein in cultivar NS was higher than that in cultivar NF (Fig. 2EF). Taken together, high levels of ROS scavenging and osmolytes and lower level of lipid peroxidation in leaves of the winter rapeseed under freezing stress would furnish a melodious redox homeostasis environment for plant growth and development, leading to the stronger freezing resistance in NS plants.

\section{Comparison of the differences in freezing tolerance between both winter rapeseed cultivars}

Numerous TFs have been reported in plants that monitor the expression of C-repeat binding transcription factor (CBF) by combining corresponding cis-elements in their promoters under cold stress. After exposed to freezing stress, CBF proteins rapidly recognize the promoter regions of downstream cold-regulated genes to activate their expression, thereby enhancing freezing tolerance (Jia et al. 2016; Zhao et al. 2016). For instance, bHLH transcription factor ICE1, calmodulinbinding transcription activator 3 (CAMTA3), brassinazole-resistant 1 (BZR1), heat shock transcription factor C1 (HSFC1), and MYB88 /MYB124 transcription factors positively regulate the CBF expression at the transcriptional level that contribute to cold stress (Ding et al. 2015; Park et al. 2015; Li et al. 2017; Xie et al. 2018). In contrast, MYB15, phytochrome interacting factor 3 (PIF3), PIF4, PIF7 and ethylene sensitivity 3 (EIN3) negatively modulate the transcriptional repression of CBF (Shi et al. 2012; Kim et al. 2017; Jiang et al. 2017). In the present study, most of AP2/ERF, WRKY, NAC, HSF, and TIFY transcription factors were up-regulated in both cultivars, while transcription factors bHLH, bZIP, ARF/GFP, OFP, and mTERF were down-regulated (Fig. 7AB; Table S6). In addition, we found that AP2/ERF and HSF genes have differences in DNA methylation levels (Table 1), indicating that these transcription factor genes may defend against freezing stress through epigenetic modifications. Interestingly, these differentially expressed TFs encoded by genes were not found at the protein level, implying that those transcriptional moderators might regulate the gene expression just at the transcription level in contribution to the freezing resistance of winter rapeseed under freezing stress. 
Table 1

The list of DNA methylation levels of some DEGs identified in both cultivars (unpublished).

\begin{tabular}{|c|c|c|c|c|c|c|c|}
\hline Gene Name & Meth.type & Meth.diff & Gene ID & $\log 2(F C)$ & $\begin{array}{l}\text { Position } \\
\text { (start) }\end{array}$ & $\begin{array}{l}\text { Position } \\
\text { (end) }\end{array}$ & Cultivar \\
\hline $\begin{array}{l}\text { Ethylene- } \\
\text { responsive } \\
\text { transcription } \\
\text { factor RAP2-4 }\end{array}$ & $\mathrm{CHH}$ & -15.1 & LOC106353462 & 2.513787538 & 66399101 & 66399300 & NTS57 \\
\hline \multirow{2}{*}{$\begin{array}{l}\text { Ethylene- } \\
\text { responsive } \\
\text { transcription } \\
\text { factor ERF114 }\end{array}$} & $\mathrm{CHH}$ & -17.4 & LOC106380120 & 3.264061601 & 11674501 & 11674700 & NTS57 \\
\hline & $\mathrm{CHH}$ & -29.3 & LOC106380120 & 3.264061601 & 11675201 & 11675400 & NTS57 \\
\hline \multirow{3}{*}{$\begin{array}{l}\text { Heat stress } \\
\text { transcription } \\
\text { factor B1 }\end{array}$} & $\mathrm{CHG}$ & 34.7 & LOC106407137 & 3.106258687 & 59711401 & 59711600 & NTS57 \\
\hline & $\mathrm{CHG}$ & 29.2 & LOC106407137 & 3.106258687 & 59711601 & 59711800 & NTS57 \\
\hline & $\mathrm{CHG}$ & 28.2 & LOC106407137 & 3.106258687 & 59711801 & 59712000 & NTS57 \\
\hline $\begin{array}{l}\text { CBL-interacting } \\
\text { serine/threonine- } \\
\text { protein kinase } 9\end{array}$ & $\mathrm{CHG}$ & -51.9 & LOC106401821 & 2.289092273 & 490801 & 491000 & NTS57 \\
\hline \multirow{2}{*}{$\begin{array}{l}\text { Caffeic acid 3-0- } \\
\text { methyltransferase }\end{array}$} & $\mathrm{CHG}$ & -27.7 & LOC106417606 & 2.739228139 & 23901201 & 23901400 & NTS57 \\
\hline & $\mathrm{CHG}$ & -28.2 & LOC106417606 & 2.739228139 & 23901301 & 23901500 & NTS57 \\
\hline $\begin{array}{l}\text { calcium- } \\
\text { dependent protein } \\
\text { kinase } 28\end{array}$ & $\mathrm{CHG}$ & 25.5 & LOC106392253 & 2.20728941 & 7242601 & 7242800 & NQF24 \\
\hline $\begin{array}{l}\text { Calcium- } \\
\text { dependent protein } \\
\text { kinase } 6\end{array}$ & $\mathrm{CHH}$ & 20.9 & LOC106400806 & 2.158244799 & 50391101 & 50391300 & NQF24 \\
\hline
\end{tabular}

Furthermore, some protein kinases including calcium-dependent protein kinases (CDPKs), CBL-interacting serine/threonine-protein kinases (CIPKs), mitogen-activated protein kinases (MAPKs), receptor-like kinases (RLKs), serine/threonine-protein kinases (SPK), and SNF1-related protein kinases (SnRKs) have been characterized to be crucial regulators of cold-stress responses in plant (Liu et al. 2017; Zhao et al. 2017; Ding et al. 2019b). In addition, a putative cold sensor chilling-tolerance divergence 1 (COLD1), was reported to mediate a cold-sensing calcium channel in rice, leading to the activation of cold-regulated genes (Ma et al. 2015; Shi et al. 2018). A similar result was obtained in the present study, a total of 7 CDPKs, 4 CIPKs, 13 MAPKs, 81 RLKs, 1 SnRK, and 42 SPKs were up-regulated in the cultivar NF under freezing stress, whereas 3 CDPKs, 3 CIPKs, 95 RLKs, 2 SnRKs, and 37 SPKs were down-regulated; a total of 9 CDPKs, 8 CIPKs, 9 MAPKs, 22 RLKs, 1 SnRK and 30 SPKs were up-regulated in the cultivar NS under freezing stress, whereas 9 CDPKs, 6 CIPKs, 11 MAPKs, 124 RLKs, 8 SnRKs and 49 SPKs were down-regulated (Table S5). Combined with the differences in DNA methylation levels of CDPK and CIPK genes (Table 1), a popular explanation is that freezing treatment might induce DNA methylation modification of these genes, thereby trigger the activation of other freezingresponsive genes, which needs further study.

Ribosomal proteins (RPs) are well-known for their role in maintaining the stability of the ribosomal complex, which performs mRNA directed protein synthesis (Moin et al. 2016). A drastic lowering of the ambient temperature entails extensive reprogramming of the gene expression pattern (He et al. 2021). In this study, compared to the control, all DAPs enriched in the ribosome pathway were accumulated in abundance in NF under freezing stress (Fig. 5B; Table S5), implying that the protein synthesis was enhanced, which might be beneficial to cope with freezing stress in NF. 
Photosynthesis is main driving force for the growth and biomass production in plants, it can supply the energy and carbon for the biosynthesis of necessary organic compounds (Nowicka et al. 2018). In this study, the photosynthesis pathway was significantly enriched by DEGs and DAPs in NS, and all ferredoxins (FDX) and chlorophyll a/b-binding proteins (CBPs) enriched in this pathway were reduced in transcription and abundance (Fig. 6; Table S5), which can recruit photons and transfer the absorbed energy towards the photosynthetic reaction centre (Palm et al. 2018), suggesting that the photosynthesis activity was crippled under freezing stress. Consistent with transcriptome and proteome results, the measured net photosynthetic rates were decreased in NS and NF under freezing stress, but it was significantly higher in the former than that in later (Fig. 1B), which might attribute to more stable chloroplasts ultrastructure in the leaves of NS.

Plant hormones, including abscisic acid (ABA), auxin, brassinosteroid (BR), cytokinin, ethylene, gibberellin, jasmonic acid (JA) and salicylic acid, combine endogenous substances with environmental signals to regulate plant growth, development and defense (Huang et al. 2017). In many instances, plants respond to environmental stresses by producing amount of ABA, ethylene and JA (Huang et al. 2016; Hu et al. 2017). Similar results were obtained in this study, a total of 115 and 104 unique DEGs in NF and NS, respectively, were involved in plant hormone signal transduction pathway (Table S5), among them, 10 and 7 DEGs were up-regulated in NF and NS, respectively, and participated in ABA signaling, encoding abscisic acid-insensitive protein (ABI), SPK and protein phosphatase 2C (PP2C), whereas 6 and 10 DEGs encoding $A B I$, SPK and abscisic acid receptor (ABR) were down-regulated in NF and NS, respectively. 5 out of 48 DEGs and 15 out of 39 DEGs were up-regulated in NF and NS, respectively, and involved in auxin signaling, encoding auxin-induced protein (AIP), auxin-responsive protein (ARP), auxin transporter protein (ATP), and GH3 auxin-responsive promoter (GH3). Therefore, we surmised that there may be more than one ABA and auxin metabolic pathway related to freezing tolerance, and one of them was enhanced. Furthermore, 14 up-regulated DEGs and 7 down-regulated DEGs were found in NF and NS, respectively, and gone in for ethylene signaling, encoding ethylene insensitive protein (EIN), EIN3-binding F-box protein (EBF), ethylene-responsive transcription factor (ERF) and ethylene receptor (ER). Correspondingly, we found that two key enzymes of ethylene biosynthesis, 1-aminocyclopropane-1-carboxylate synthases (ACS) and 1-aminocyclopropane-1carboxylate oxidases (ACO), were up-regulated in NF and down-regulated in NS, this may be due to feedback regulation in NS, resulting in the decreasing of ethylene metabolism. 5 DEGs were up-regulated in NF and engaged in JA signaling, encoding TIFY proteins and transcription factor MYC2, which was a key regulator for the activation of JA under cold stress (Kazan, 2015). These are consistent with what has been found in the previous research, which indicated that high levels of plant hormones could improve the freezing resistance (Pu et al. 2019; Wei et al. 2021). In addition, one DEG was down-regulated in NF, encoding protein BZR, which is plant-specific positive regulator of BR signaling (Li et al. 2017). BRs can be perceived by the brassinosteroid insensitive1 (BRI1; She et al. 2011), and BRI1 interacted with BRI1-associated receptor kinase and positively regulated BZR1 (Fang et al. 2019). Interestingly, in this study, 2 BRI1 genes were found and they were down-regulated in NF, however, we found only one down-regulated BZR2 gene in NS. Therefore, we speculate that BZR1 could combine with BRI1 to negatively regulate BR signaling in NF under freezing stress. Apart from that, numerous studies have reported that BRs could improve photosynthesis and ROS scavenging enzymes activities (Xia et al. 2018; Yan et al. 2020). Overall these findings are in accordance with the results of photosynthesis and antioxidant enzymes (Fig. 1B; Fig. 2). Simultaneously, other DEGs identified in both cultivars in our study mainly represent some TFs, which are essential for plants under abiotic stresses (Li et al. 2019; Frank et al. 2020). Taken together, these results suggested that plant hormones play profound roles in coping with the freezing stress of rapeseed.

Plant lipoxygenases (LOXs) are a kind of multifunctional fatty acid dioxygenases that catalyze the peroxidation of linolenic acid and linoleic acid. The synthesis of jasmonic acid begins with the peroxidation of a-linolenic acid by lipoxygenase. It was reported that biotic and abiotic stresses could cause the liberation of a-linolenic acid, which is an important precursor for the biosynthesis of jasmonic acid (Yan et al. 2013; Zhao et al. 2014), which interacted with other hormones signaling to regulate leaf tolerance to cold stress (Hu et al. 2017). In this study, all unique LOXs participated in the alpha-linolenic acid metabolism were found to be down-regulated in NF, whereas they were up-regulated in NS (Fig. 5A; Table S5). An interesting finding was that the alpha-linolenic acid metabolism was enriched by DAPs in NF, and all DAPs

Page $10 / 25$ 
were accumulated in abundance (Fig. 5B; Table S5). These results suggested that there are differences in temporal and spatial between the expression of gene and protein. Beyond those, combined with JA signaling resulting from DEGs, implying that increased jasmonic acid signal might regulate the expression of downstream freezing-responsive genes.

Plant secondary metabolites often have no fundamental role in the maintenance of plant life processes, but they are crucial for the plant to interrelate to its environment for adaptation and defense (Ramakrishna et al. 2011). In the present study, there were 7 and 6 unique up-regulated DEGs in NF and NS, respectively, encoding caffeic acid O-methyltransferase (CCMT), shikimate O-hydroxycinnamoyltransferase (SHCT) and S-adenosylmethionine-dependent methyltransferase (SAMTs), implicated in the secondary metabolism (Fig. 5B; Table S5), which were proclaimed in many plant species (Ranjan et al. 2020). It is in agreement with those reported by Zeng et al. (2018) and Pu et al. (2019). It is interesting to note that some DNA methylation differences in the CCMT gene were found under freezing inducement (Table 1), which is particularly important when investigating the epigenetic freezing-reponsive mechanism of winter rapeseed. A further novel finding was that the plant-pathogen interaction pathway was significantly enriched by DEGs in NS, and most of DEGs enriched in this pathway were up-regulated (Fig. 6A; Table S5), implying that its defense system was strengthen after freezing treatment. These results suggested that accumulated secondary metabolites and enhanced plant-pathogen interaction together contribute to the formation of freezing tolerance of rapeseed cultivar NS under freezing stress.

It is acknowledged that heat shock proteins (HSPs) regarded as the target genes of HSFs, are beneficial to defense against cold stress in grasses (Wang et al. 2016). Similarly, Lin et al. (2019) reported that some HSPs and chaperone proteins (CPS) were induced by cold stress. Scarpeci et al. (2008) indicated that ROS through direct interacted with HSFs to offer abiotic stress tolerance in plants. In the present study, some up-regulated HSFs, CPs and HSPs were concomitant with high level of POD activity (Fig. 2C; Table S5), showing that the HSFs might interact with ROS signals to regulate the expression of CPs and HSPs under freezing stress. Furthermore, several up-regulated DEGs encoding protein disulfide isomerase (PDI) associated with protein synthesis were found in NS, whereas they were down-regulated in NF (Table S5). In addition, we discovered 10 unique down-regulated DEGs in NF, which encode mitochondria-localized ATP synthase subunit (ASS) and cytochrome c oxidase (CCO), and 2 unique up-regulated CCOs in NS (Table S5). As we all know, ASS is the structural basis of proton transportation and energy generation in mitochondrial (Klusch et al. 2017). CCO is the last respiratory complex of the electron transmit chain in mitochondria and is responsible for transferring electrons to the final acceptor oxygen in the respiratory metabolism (Dahan et al. 2014). The down-regulated ASS and CCO indicated the decrease of energy requirement in the developing leaf of NF under freezing stress, which might be one of the major reasons why it was more freezing-sensitive than the cultivar NS.

\section{A possible freezing-responsive molecular network in developing winter rapeseed leaves}

In this study, a freezing-responsive molecular network was produced in developing rapeseed leaves based on the results of transcriptome and proteome (Fig. 8). This network includes three important functional components: firstly, rapeseed plants leaves heighten $\mathrm{Ca}^{2+}$ signal pathway by up-regulation of CDPK, CIPK, MAPK, RLK, SnRK and SPK under freezing stress; subsequently, these up-regulated protein kinases genes corporate with TFs to trigger the expression of downstream freezing-tolerant genes; lastly, those activating genes cause some changes in metabolic pathways (enhanced plant hormones biosynthesis, ROS balancing and plant-pathogen interaction, accelerated biosynthesis of proteins and secondary metabolites, decreased photosynthesis), showing that the production of plant hormones, antioxidation enzymes and secondary metabolites might be positively contributed to freezing tolerance.

\section{Conclusions}

After freezing treatment, rapeseed cultivar more tolerant to freezing stress would fabricate stronger signals $\left(\mathrm{Ca}^{2+}, \mathrm{ROS}^{2}\right.$, ABA, Auxin, BR, Ethylene and JA), metabolic pathways (protein biosynthesis, plant-pathogen interaction and secondary 
metabolites synthesis) and ROS scavenging capacity, more osmolytes (soluble sugar and protein) and lower level of lipid peroxidation in leaves, as well as more stable ultrastructure.

\section{Abbreviations}

ABA, bscisic acid;

ACO, 1-aminocyclopropane-1-carboxylate oxidase;

ACS, 1-aminocyclopropane-1-carboxylate synthase;

ABR, abscisic acid receptor;

ARP, auxin responsive protein;

ASS, ATP synthase subunit;

ATP, auxin transporter protein;

BR, brassinosteroid;

$\mathrm{BRI}$, brassinosteroid insensitive;

BZR, brassinazole-resistant;

CAT, catalase;

CCMT, caffeic acid 0-methyltransferase;

CCO, cytochrome c oxidase;

CDPK, $\mathrm{Ca}^{2+}$-dependent protein kinase;

CIPK, CBL-interacting serine/threonine-protein kinase;

$\mathrm{CP}$, chaperone protein;

DAP, differentially abundant protein;

DDA, data-dependent acquisition;

DEG, differentially expressed gene;

DIA, data-independent acquisition;

EIN, ethylene insensitive protein;

ERF, ethylene response factor;

FPKM, fragments per kilobase per million;

GH3, GH3 auxin-responsive promoter;

GO, Gene Ontology; 
HSF, heat stress transcription factor;

HSP, heat shock protein;

JA, jasmonic acid;

KEGG, Kyoto Encyclopedia of Genes and Genomes;

LOX, lipoxygenases;

MAPK, mitogen-activated protein kinase;

MDA, malondialdehyde;

PCA, principal component analysis;

PIF, phytochrome interacting factor;

POD, peroxidase;

PP2C, protein phosphatase 2C;

RLK, receptor-like kinase;

ROS, reactive oxygen species;

SAMT, S-adenosylmethionine-dependent methyltransferase;

SHCT, shikimate O-hydroxycinnamoyltransferase;

SnRK, SNF1-related protein kinase;

SOD, superoxide dismutase;

SPK, serine/threonine-protein kinase;

TEM, transmission electron microscope;

TF, transcription factor;

Table S1. The proteins database of Brassica napus downloaded from the NCBI, and the list of all genes and proteins identified in transcriptome and proteome, respectively;

Table S2. DEGs identified in leaves between the stressed and control rapeseed cultivar NF and NS, respectively;

Table S3. DAPs identified in leaves between the stressed and control rapeseed cultivar NF and NS, respectively;

Table S4. DEGs and DAPs jointly owned in NFC_NFT and NSC_NST;

Table S5. The unique DEGs and DAPs in NFC_NFT and NSC_NST;

Table S6. Differentially expressed TFs identified in leaves between the control and the stressed rapeseed cultivar NF and NS, respectively, by transcriptome. 


\section{Declarations}

\section{Author Contributions}

This work presented here was finished by all authors. J.W. performed the multi-omics joint analysis and wrote the manuscript; G.Z. executed the physiological and biochemical assays; X.D. and S.L. participated in the proteomic data analysis; H.L. and Y.W. cultivated all plant samples; Z.L. designed the experiments and revised the paper. All authors have read and approved the final manuscript.

\section{Conflict Interests}

The authors declare no competing financial interests.

\section{Acknowledgments}

The authors gratefully acknowledge the partial financial support from the Scientific Research Start-up Funds for Openlyrecruited Doctors, Science and Technology Innovation Funds of Gansu Agricultural University (GAU-KYQD-2019-25), Ministry of Science and Technology of China (2018YFD0100500), National Natural Science Foundation of China (31660404) and the Special Funds for the Central Government to Guide Local Technological Development of China (ZCYD-2020-2-3).

\section{References}

1. Ding Y, Shi Y, Yang S (2019a) Advances and challenges in uncovering cold tolerance regulatory mechanisms in plants. New Phytol 222(4):1690-1704

2. Shi Y, Ding Y, Yang S (2018) Molecular regulation of CBF signaling in cold acclimation. Trends Plant Sci 23:623-637

3. Xu Y, Zeng X, Wu J, Zhang F, Li C, Jiang J, Wang Y, Sun W (2018) iTRAQ-based quantitative proteome revealed metabolic changes in winter turnip rape (Brassica rapa L.) under cold stress. Int J Mol Sci 19(11):3346

4. Ding Y, Li H, Zhang X, Xie Q, Gong Z, Yang S (2015) OST1 kinase modulates freezing tolerance by enhancing ICE1 stability in arabidopsis. Dev Cell 32:278-289

5. Zhang CY, Zhang ZY, Li JH, Li F, Liu HH, Yang WS, Chong K, Xu YY (2017) OsMAPK3 phosphorylates OsbHLH002/OsICE1 and inhibits its ubiquitination to activate OsTPP1 and enhances rice chilling tolerance. Dev Cell 43:731-743

6. Zeng X, Xu Y, Jiang J, Zhang F, Ma L, Wu D, Wang Y, Sun W (2018) iTRAQ-based comparative proteomic analysis of the roots of two winter Turnip Rapes (Brassica rapa L.) with different freezing-tolerance. Int J Mol Sci 19(12):4077

7. Pu Y, Liu L, Wu J, Zhao Y, Bai J, Ma L, Yue J, Jin J, Niu Z, Fang Y, Sun W (2019) Transcriptome profile analysis of winter rapeseed (Brassica napus L.) in response to freezing stress, reveal potentially connected events to freezing stress. Int J Mol Sci 20(11):2771

8. Egertson JD, Kuehn A, Merrihew GE, Bateman NW, MacLean BX, Ting YS, Canterbury JD, Marsh DM, Kellmann M, Zabrouskov V, Wu CC, MacCoss MJ (2013) Multiplexed MS/MS for improved data-independent acquisition. Nat Methods 10(8):744-756

9. Wei J, Zheng G, Yu X, Liu S, Dong X, Cao X, Fang X, Li H, Jin J, Mi W, Liu Z (2021) Comparative transcriptomics and proteomics analyses of leaves reveals a freezing stress-responsive molecular network in winter rapeseed (Brassica rapa L.). Front Plant Sci 12:664311

10. Ma N, Hu C, Wan L, Hu Q, Xiong J, Zhang C (2017) Strigolactones improve plant growth, photosynthesis, and alleviate oxidative stress under salinity in rapeseed (Brassica napus L.) by regulating gene expression. Front Plant Sci 8:1671 
11. Toyooka K, Sato M, Kutsuna N, Nagata N (2014) Development of high resolution TEM image acquisition system by using high-pressure freezing method. Plant Morphology 26:3-8

12. Chen MX, Zhu FY, Wang FZ, Ye NH, Gao B, Chen X, Zhao SS, Fan T, Cao YY, Liu TY, Su ZZ, Xie LJ, Hu QJ, Wu HJ, Xiao S, Zhang J, Liu YG (2019) Alternative splicing and translation play important roles in hypoxic germination in rice. J Exp Bot 70(3):817-833

13. Wei J, Liu X, Li L, Zhao H, Liu S, Yu X, Shen Y, Zhou Y, Zhu Y, Shu Y, Ma H (2020) Quantitative proteomic, physiological and biochemical analysis of cotyledon, embryo, leaf and pod reveals the effects of high temperature and humidity stress on seed vigor formation in soybean. BMC Plant Biol 20:127

14. Song L, Liu Z, Tong J, Xiao L, Ma H, Zhang H (2015) Comparative proteomics analysis reveals the mechanism of fertility alternation of thermosensitive genic male sterile rice lines under low temperature inducement. Proteomics 15(11):1884-1905

15. Kim D, Pertea G, Trapnell C, Pimentel H, Kelley R, Salzberg SL (2013) TopHat2: accurate alignment of transcriptomes in the presence of insertions, deletions and gene fusions. Genome Biol 14(4):R36

16. Trapnell C, Williams BA, Pertea G, Mortazavi A, Kwan G, van Baren MJ, Salzberg SL, Wold BJ, Pachter L (2010) Transcript assembly and quantification by RNA-Seq reveals unannotated transcripts and isoform switching during cell differentiation. Nat Biotechnol 28:511-515

17. Bruderer R, Bernhardt OM, Gandhi T, Miladinović SM, Cheng LY, Messner S, Ehrenberger T, Zanotelli V, Butscheid Y, Escher C, Vitek O, Rinner O, Reiter L (2015) Extending the limits of quantitative proteome profiling with dataindependent acquisition and application to acetaminophen-treated three-dimensional liver microtissues. Mol Cell Proteomics 14(5):1400-1410

18. Ma J, Chen T, Wu S, Yang C, Bai M, Shu K, Li K, Zhang G, Jin Z, He F, Hermjakob H, Zhu Y (2019a) iProX: an integrated proteome resource. Nucleic Acids Res 47(1): 1211-1217

19. Binns D, Dimmer E, Huntley R, Barrell D, O'Donovan C, Apweiler R (2009) QuickGO: a web-based tool for Gene Ontology searching. Bioinformatics 25(22):3045-3046

20. Kanehisa M, Sato Y, Kawashima M, Furumichi M, Tanabe M (2016) KEGG as a reference resource for gene and protein annotation. Nucleic Acids Res 44(1):457-462

21. Zhu J (2016) Review abiotic stress signaling and responses in plants. Cell 167:313-324

22. Guo XY, Liu DF, Chong K (2018) Cold signaling in plants: insights into mechanisms and regulation. J Integr Plant Biol 60:745-756

23. Ma X, Chen C, Yang M, Dong X, Lv W, Meng Q (2018) Cold-regulated protein (SICOR413IM1) confers chilling stress tolerance in tomato plants. Plant Physiol Biochem 124:29-39

24. Baxter A, Mittler R, Suzuki N (2014) ROS as key players in plant stress signalling. J Exp Bot 65:1229-1240

25. Ray PD, Huang BW, Tsuji Y (2012) Reactive oxygen species (ROS) homeostasis and redox regulation in cellular signaling. Cell Signal 24:981-990

26. Ma L, Coulter JA, Liu L, Zhao Y, Chang Y, Pu Y, Zeng X, Xu Y, Wu J, Fang Y, Bai J, Sun W (2019b) Transcriptome analysis reveals key cold-stress-responsive genes in winter rapeseed (Brassica rapa L.). Int J Mol Sci 20(5): 1071

27. Deng S, Sun J, Zhao R, Ding M, Zhang Y, Sun Y, Wang W, Tan Y, Liu D, Ma X, Hou P, Wang M, Lu C, Shen X, Chen S (2015) Populus euphratica APYRASE2 enhances cold tolerance by modulating vesicular trafficking and extracellular ATP in arabidopsis plants. Plant Physiol 169(1):530-548

28. Jia Y, Ding Y, Shi Y, Zhang X, Gong Z, Yang S (2016) The cbfs triple mutants reveal the essential functions of CBFs in cold acclimation and allow the definition of CBF regulons in arabidopsis. New Phytol 212:345-353

29. Zhao C, Zhang Z, Xie S, Si T, Li Y, Zhu JK (2016) Mutational evidence for the critical role of CBF transcription factors in cold acclimation in arabidopsis. Plant Physiol 171:2744-2759

Page $15 / 25$ 
30. Park S, Lee CM, Doherty CJ, Gilmour SJ, Kim Y, Thomashow MF (2015) Regulation of the arabidopsis CBF regulon by a complex low temperature regulatory network. Plant J 82:193-207

31. Li H, Ye K, Shi Y, Cheng J, Zhang X, Yang S (2017) BZR1 positively regulates freezing tolerance via CBF-dependent and CBF-independent pathways in arabidopsis. Mol Plant 10:545-559

32. Xie Y, Chen P, Yan Y, Bao C, Li X, Wang L, Shen X, Li H, Liu X, Niu C, Zhu C, Fang N, Shao Y, Zhao T, Yu J, Zhu J, Xu L, van Nocker S, Ma F, Guan Q (2018) An atypical R2R3 MYB transcription factor increases cold hardiness by CBFdependent and CBF-independent pathways in apple. New Phytol 218(1):201-218

33. Shi Y, Tian S, Hou L, Huang X, Zhang X, Guo H, Yang S (2012) Ethylene signaling negatively regulates freezing tolerance by repressing expression of CBF and type-A ARR genes in arabidopsis. Plant Cell 24:2578-2595

34. Kim SH, Kim HS, Bahk S, An J, Yoo Y, Kim JY, Chung WS (2017) Phosphorylation of the transcriptional repressor MYB15 by mitogen-activated protein kinase 6 is required for freezing tolerance in arabidopsis. Nucleic Acids Res 45(11):6613-6627

35. Jiang B, Shi Y, Zhang X, Xin X, Qi L, Guo H, Li J, Yang S (2017) PIF3 is a negative regulator of the CBF pathway and freezing tolerance in Arabidopsis. Proc Natl Acad Sci 114(32):6695-6702

36. Liu Z, Jia Y, Ding Y, Shi Y, Li Z, Guo Y, Gong Z, Yang S (2017) Plasma membrane CRPK1-mediated phosphorylation of 14-3-3 proteins induces their nuclear import to fine-tune CBF signaling during cold response. Mol Cell 66:117-128

37. Zhao C, Wang P, Si T, Hsu CC, Wang L, Zayed O, Yu Z, Zhu Y, Dong J, Tao WA, Zhu JK (2017) MAP kinase cascades regulate the cold response by modulating ICE1 protein stability. Dev Cell 43:618-629

38. Ding Y, Lv J, Shi Y, Gao J, Hua J, Song C, Gong Z, Yang S (2019b) EGR2 phosphatase regulates OST1 kinase activity and freezing tolerance in arabidopsis. EMBO J 38: e99819

39. Ma Y, Dai X, Xu Y, Luo W, Zheng X, Zeng D, Pan Y, Lin X, Liu H, Zhang D (2015) COLD1 confers chilling tolerance in rice. Cell 160:1209-1221

40. Moin M, Bakshi A, Saha A, Dutta M, Madhav SM, Kirti PB (2016) Rice ribosomal protein large subunit genes and their spatio-temporal and stress regulation. Front Plant Sci 7:1284

41. He CG, Gualerzi CO (2021) The ribosome as a switchboard for bacterial stress response. Front Microbiol 11:619038

42. Nowicka B, Ciura J, Szymańska R, Kruk J (2018) Improving photosynthesis, plant productivity and abiotic stress tolerance current trends and future perspectives. Plant Physiol 231:415-433

43. Palm DM, Agostini A, Averesch V, Girr P, Werwie M, Takahashi S, Satoh H, Jaenicke E, Paulsen H (2018) Chlorophyll a/b binding-specificity in water-soluble chlorophyll protein. Nat Plants 4(11):920-929

44. Huang H, Liu B, Liu L, Song S (2017) Jasmonate action in plant growth and development. J Exp Bot 68(6):13491359

45. Huang YC, Niu CY, Yang CR, Jinn TL (2016) The heat stress factor HSFA6b connects ABA signaling and ABAmediated heat responses. Plant Physiol 172(2):1182-1199

46. Hu Y, Jiang Y, Han X, Wang H, Pan J, Yu D (2017) Jasmonate regulates leaf senescence and tolerance to cold stress: crosstalk with other phytohormones. J Exp Bot 68(6):1361-1369

47. Kazan K (2015) Diverse roles of jasmonates and ethylene in abiotic stress tolerance. Trends Plant Sci 20(4):219-229

48. She J, Han Z, Kim TW, Wang J, Cheng W, Chang J, Shi S, Wang J, Yang M, Wang ZY, Chai J (2011) Structural insight into brassinosteroid perception by BRI1. Nature 474:472-476

49. Fang P, Yan M, Chi C, Wang M, Zhou Y, Zhou J, Shi K, Xia X, Foyer CH, Yu J (2019) Brassinosteroids act as a positive regulator of photoprotection in response to chilling stress. Plant Physiol 180(4):2061-2076

50. Xia XJ, Fang PP, Guo X, Qian XJ, Zhou J, Shi K, Zhou YH, Yu JQ (2018) Brassinosteroid-mediated apoplastic H2O2glutaredoxin 12/14 cascade regulates antioxidant capacity in response to chilling in tomato. Plant Cell Environ 41:1052-1064

Page $16 / 25$ 
51. Yan MY, Xie DL, Cao JJ, Xia XJ, Shi K, Zhou YH, Zhou J, Foyer CH, Yu JQ (2020) Brassinosteroid-mediated reactive oxygen species are essential for tapetum degradation and pollen fertility in tomato. Plant J 102(5):931-947

52. Li B, Liu Y, Cui XY, Fu JD, Zhou YB, Zheng WJ, Lan JH, Jin LG, Chen M, Ma YZ, Xu ZS, Min DH (2019) Genome-wide characterization and expression analysis of soybean TGA transcription factors identified a novel TGA gene involved in drought and salt tolerance. Front Plant Sci 10:549

53. Frank M, Cortleven A, Novák O, Schmülling T (2020) Root-derived trans-zeatin cytokinin protects Arabidopsis plants against photoperiod stress. Plant Cell Environ 43(11):2637-2649

54. Yan L, Zhai Q, Wei J, Li S, Wang B, Huang T, Du M, Sun J, Kang L, Li CB, Li C (2013) Role of tomato lipoxygenase D in wound-induced jasmonate biosynthesis and plant immunity to insect herbivores. PLoS Genet 9(12):e1003964

55. Zhao Y, Dong W, Zhang N, Ai X, Wang M, Huang Z, Xiao L, Xia G (2014) A wheat allene oxide cyclase gene enhances salinity tolerance via jasmonate signaling. Plant Physiol 164(2):1068-1076

56. Ramakrishna A, Ravishankar GA (2011) Influence of abiotic stress signals on secondary metabolites in plants. Plant Signal Behav 6(11):1720-1731

57. Ranjan R, Kumar N, Gautam A, Kumar Dubey A, Pandey SN, Mallick S (2020) Chlorella sp. modulates the glutathione mediated detoxification and S-adenosylmethionine dependent methyltransferase to counter arsenic toxicity in Oryza sativa L. Ecotoxicol Environ Saf 208:111418

58. Wang Y, Dai Y, Tao X, Wang JZ, Cheng HY, Yang H, Ma XR (2016) Heat shock factor genes of tall fescue and perennial ryegrass in response to temperature stress by RNA-Seq analysis. Front Plant Sci 6:1226

59. Lin Q, Xie Y, Guan W, Duan Y, Wang Z, Sun C (2019) Combined transcriptomic and proteomic analysis of cold stress induced sugar accumulation and heat shock proteins expression during postharvest potato tuber storage. Food Chem 297:124991

60. Scarpeci TE, Zanor MI, Valle EM (2008) Investigating the role of plant heat shock proteins during oxidative stress. Plant Signal Behav 3:856-857

61. Klusch N, Murphy BJ, Mills DJ, Yildiz O, Kuhlbrandt W (2017) Structural basis of proton translocation and force generation in mitochondrial ATP synthase. Elife 6:e33274

62. Dahan J, Tcherkez G, Macherel D, Benamar A, Belcram K (2014) Disruption of the CYTOCHROME C OXIDASE DEFICIENT1 gene leads to cytochrome c oxidase depletion and reorchestrated respiratory metabolism in arabidopsis. Plant Physiol 166:1788-1802

\section{Figures}



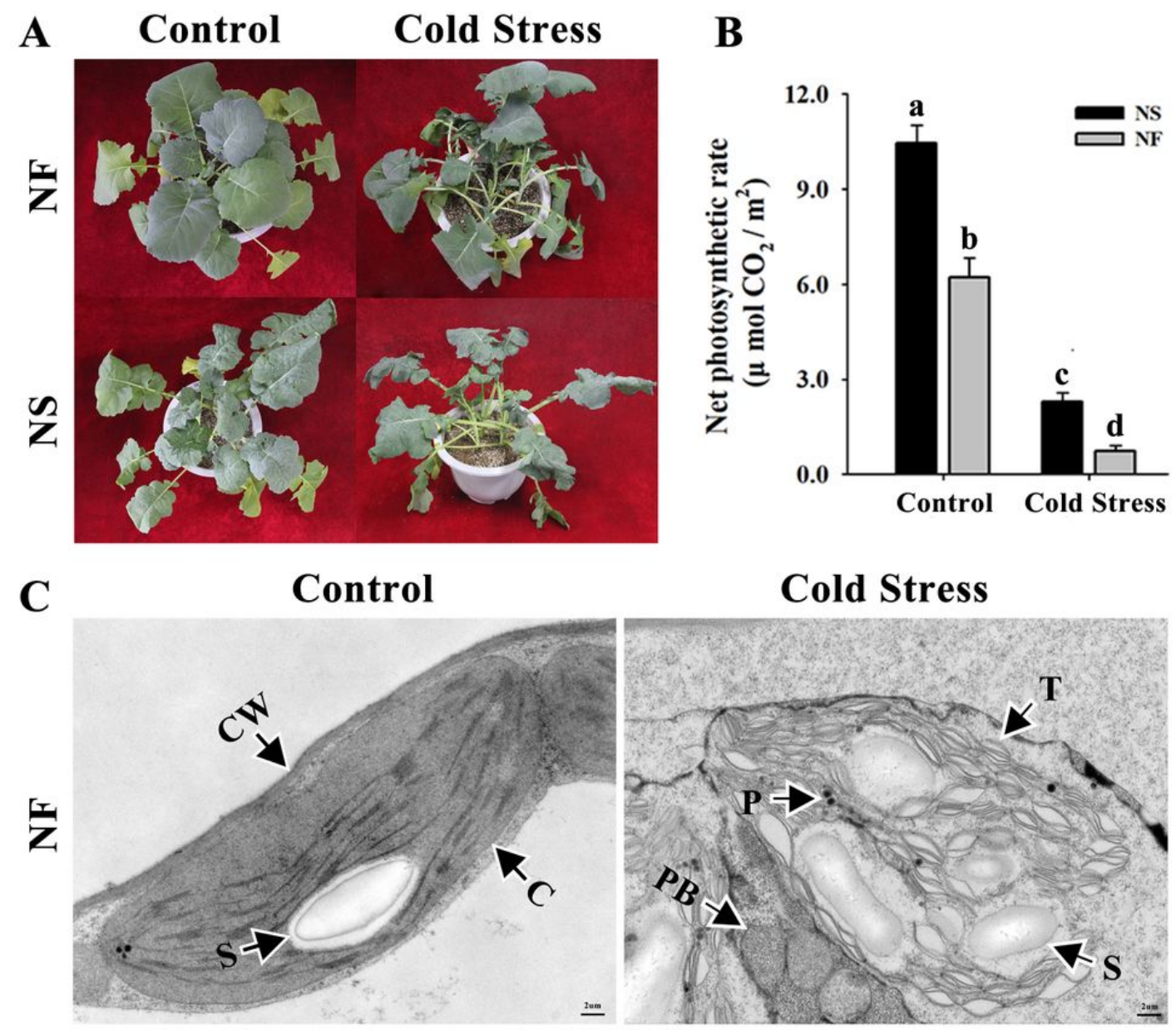

\section{Cold Stress}

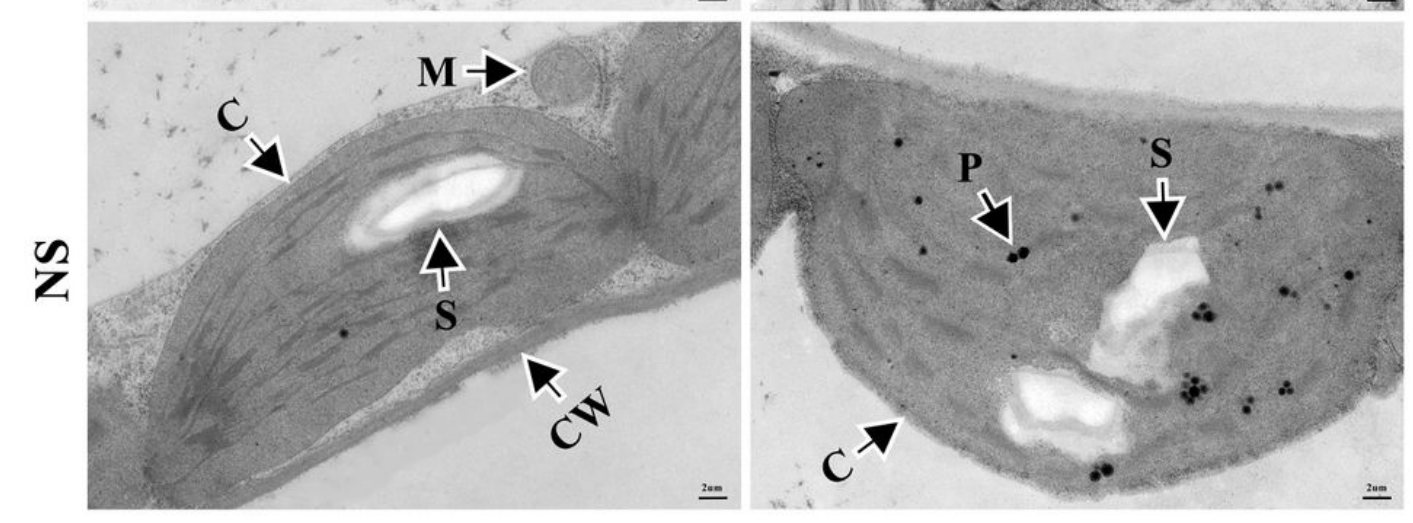

Figure 1

Phenotype, microstructure and photosynthesis changes in leaves of winter rapeseed under freezing treatment $(A)$, Illustration of 5-week-old winter rapeseed plants after freezing treatment. (B), The net photosynthetic rates of both winter rapeseed cultivars after freezing treatment. P-values are means \pm SD from three biological replicates $(p<0.05)$. (C), Ultrastructure changes in both winter rapeseed cultivars after freezing treatment for $24 \mathrm{~h}$ and control, respectively. $\mathrm{C}$, chloroplast; CW, cell wall; M, mitochondrion; P, plastoglobulus; PB, protein body; S, starch grain; T, thylakoid. 

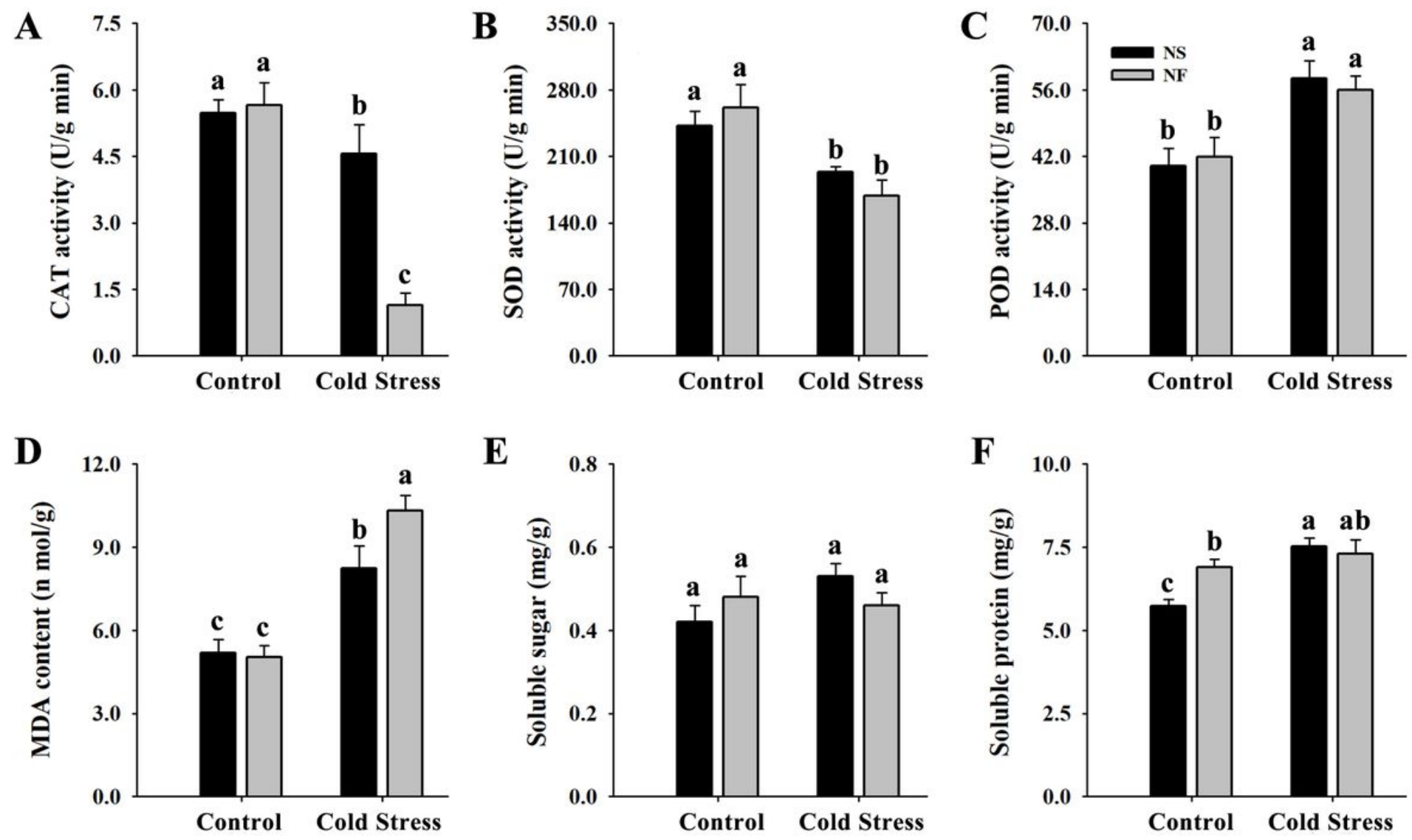

Figure 2

The physiochemical changes in leaves of winter rapeseed under freezing treatment. (A), CAT activity; (B), SOD activity; (C), POD activity; (D), MDA content; (E), Soluble sugar content; $(F)$, Soluble protein content; P-values are means $\pm S D$ from three biological replicates $(\mathrm{p}<0.05)$. 

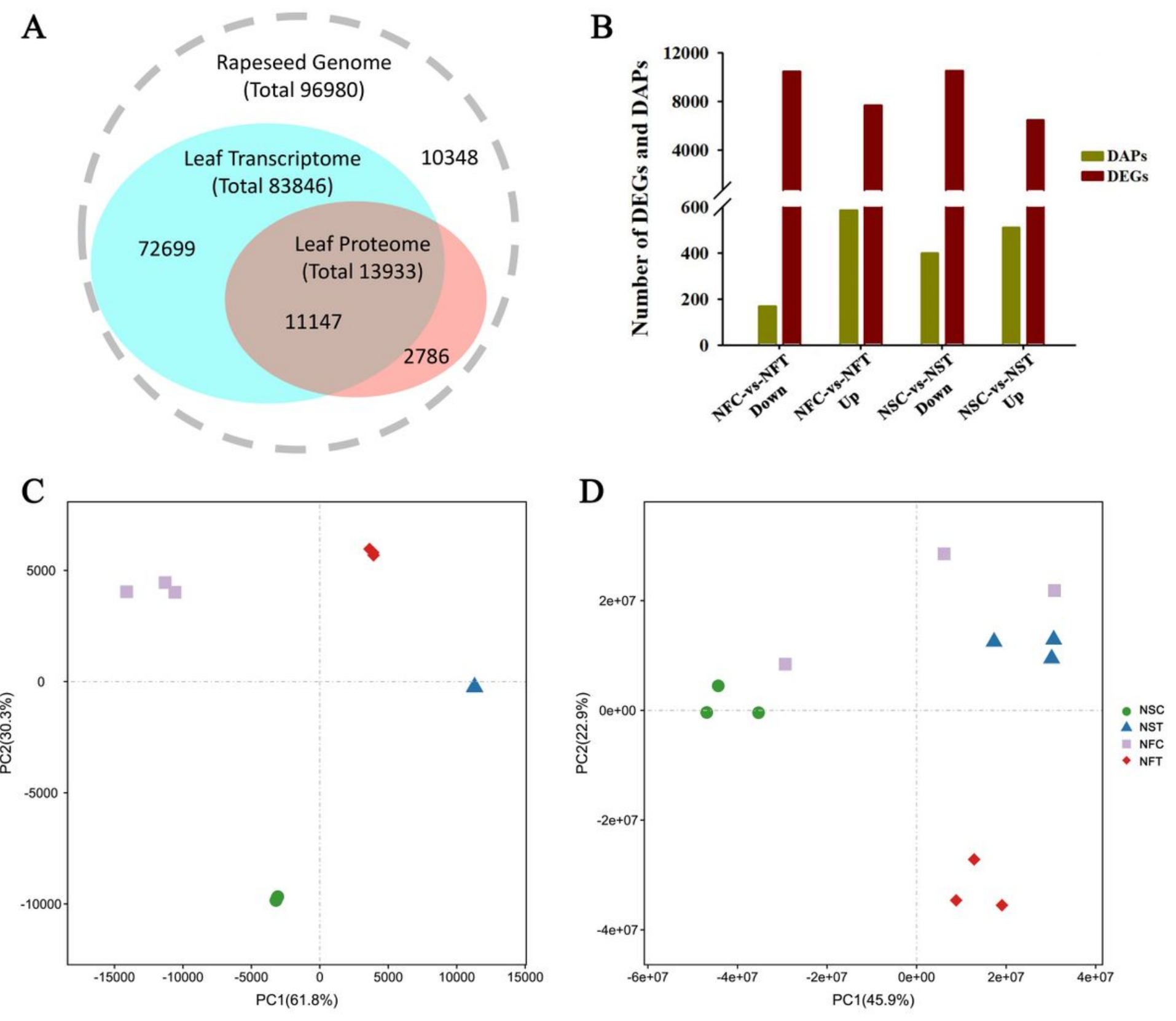

Figure 3

The overview of transcriptome and proteome in leaves of winter rapeseed. (A), Congruency between the detected transcripts and proteins in leaves of both cultivars; (B), The number of identified DEGs and DAPs; The PCA of detected genes (C) and proteins (D) by the RNA sequencing and DIA quantification, respectively. 

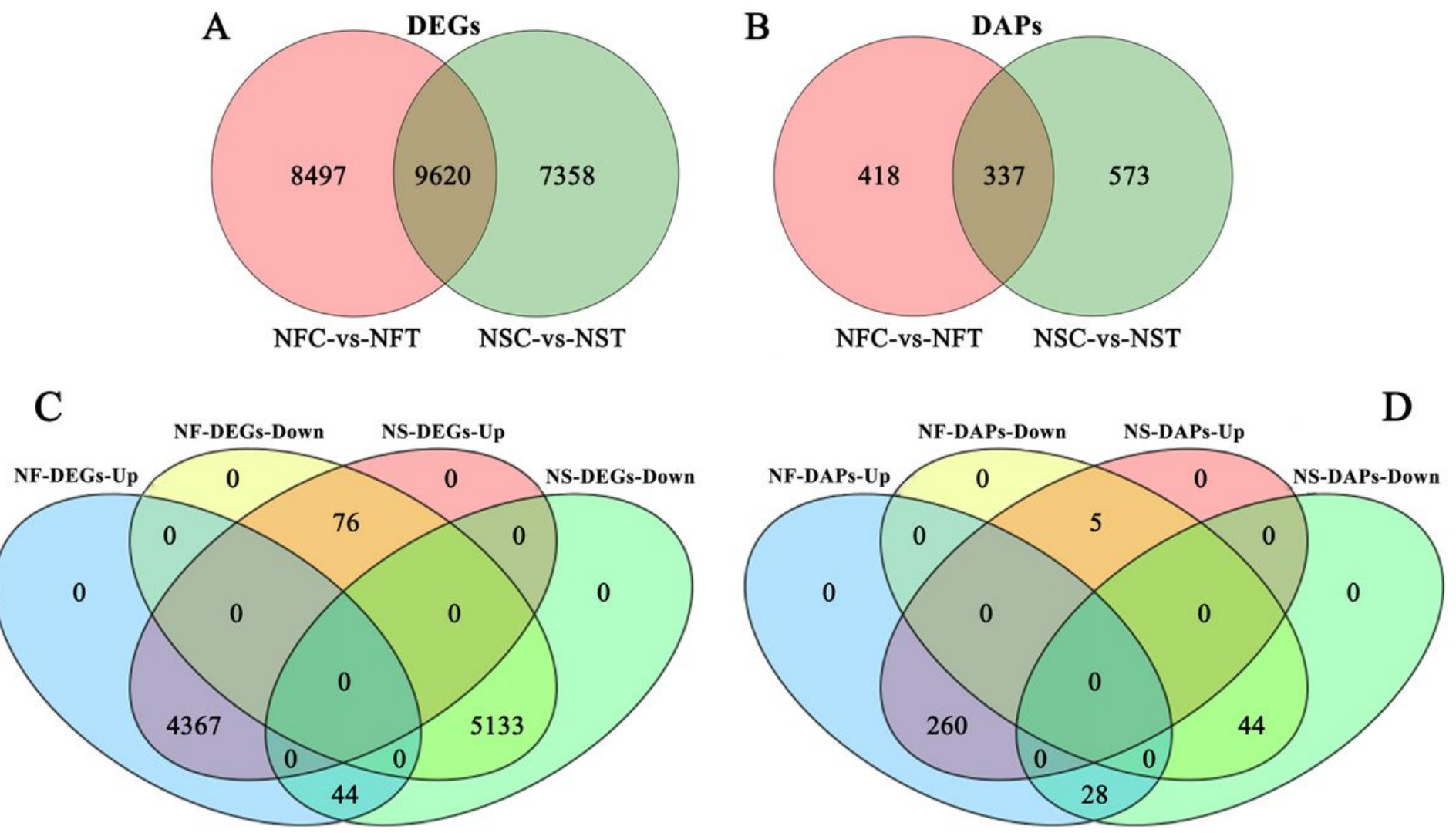

\section{Figure 4}

Comparison of transcripts and proteins abundance in developing winter rapeseed leaves. Venn diagram of identified DEGs (A) and DAPs (B) in leaves of both cultivars, respectively; (C), Venn diagram of shared DEGs (C) and DAPs (D) in leaves of both cultivars, respectively.

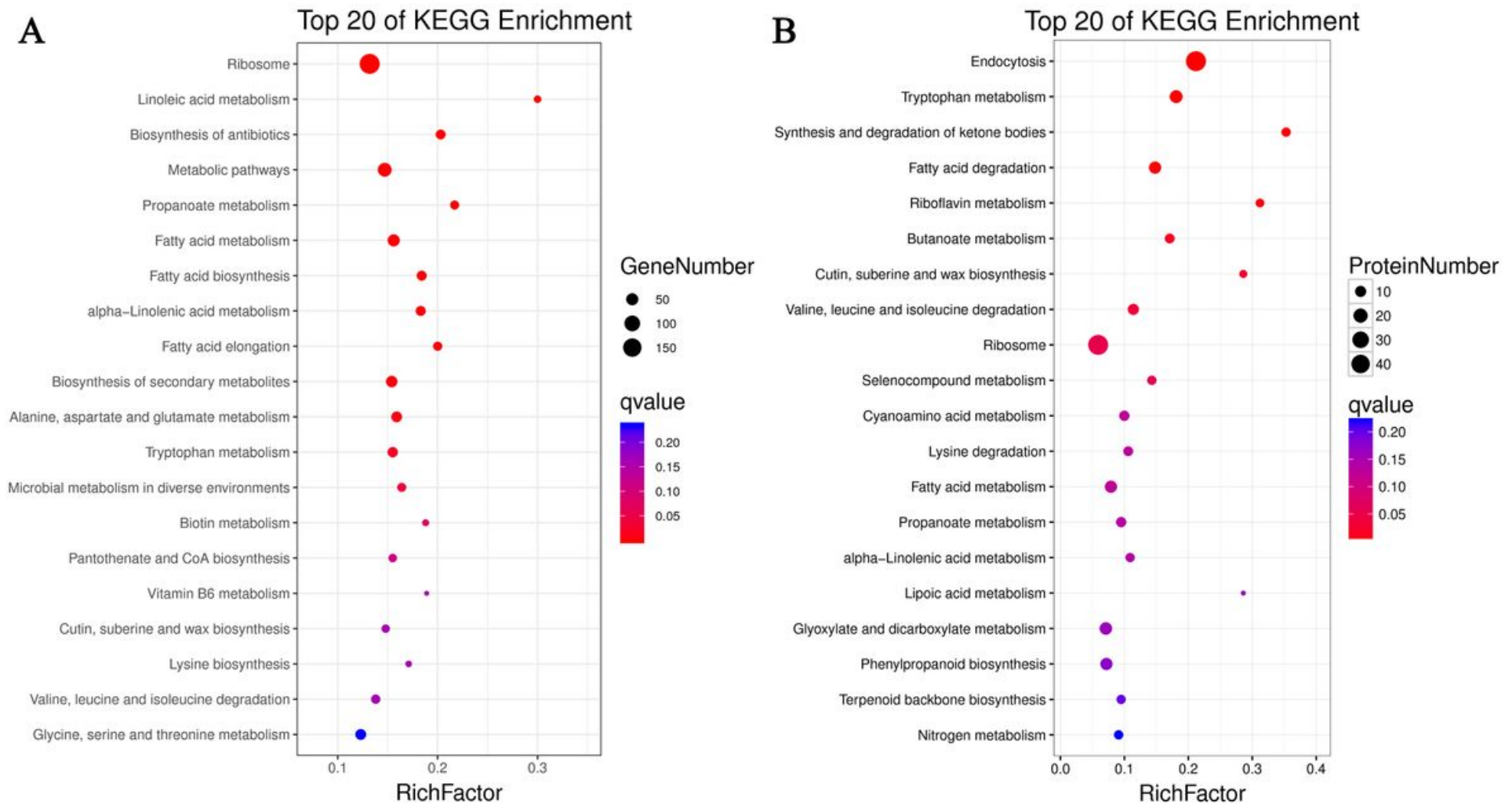


Figure 5

KEGG enrichment analysis. Top 20 KEGG pathways enriched by unique DEGs (A) and DAPs (B), respectively, in winter rapeseed cultivar NF after freezing treatment.

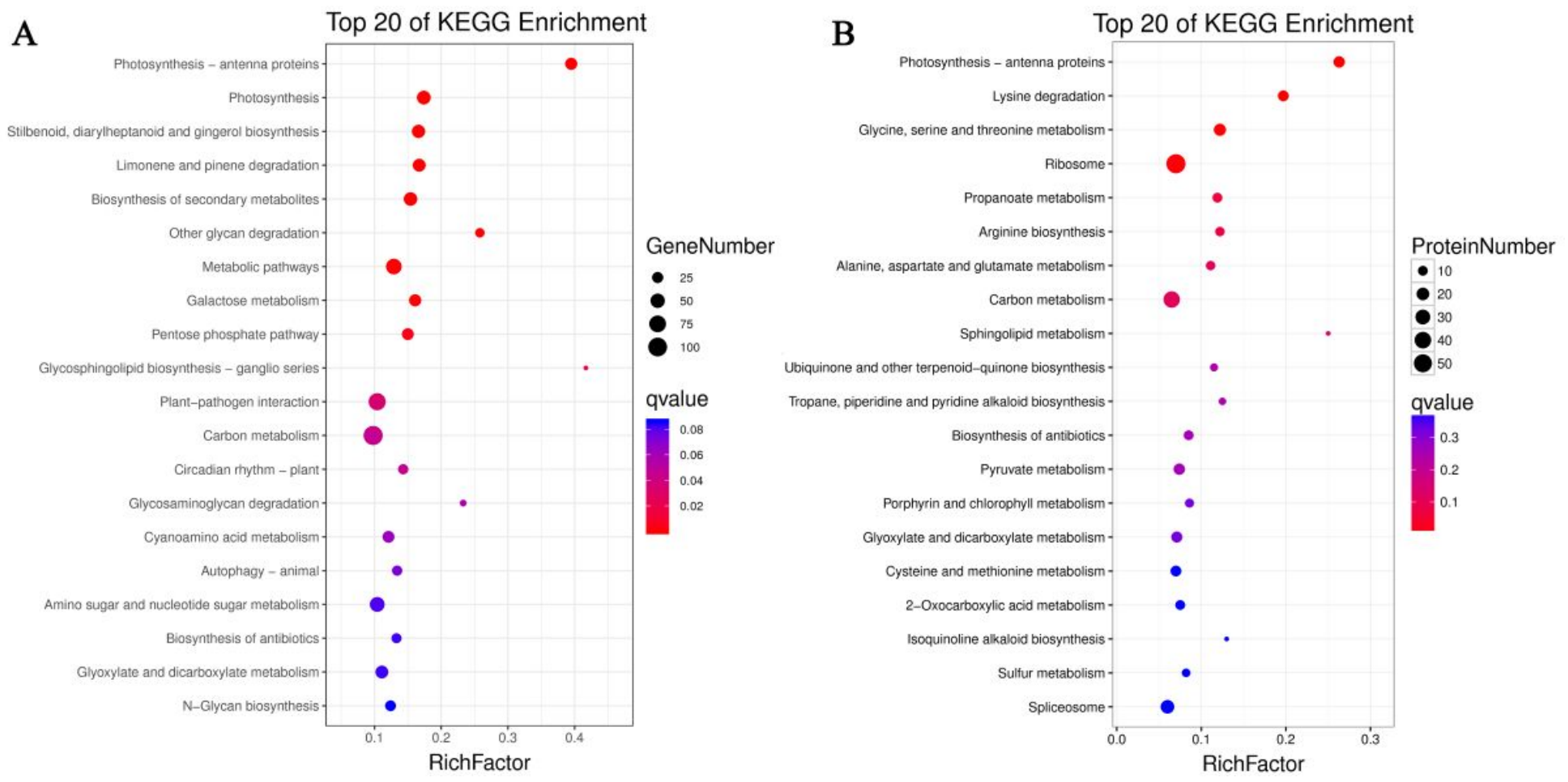

\section{Figure 6}

KEGG enrichment analysis. Top 20 KEGG pathways enriched by unique DEGs (A) and DAPs (B), respectively, in winter rapeseed cultivar NS after freezing treatment. 


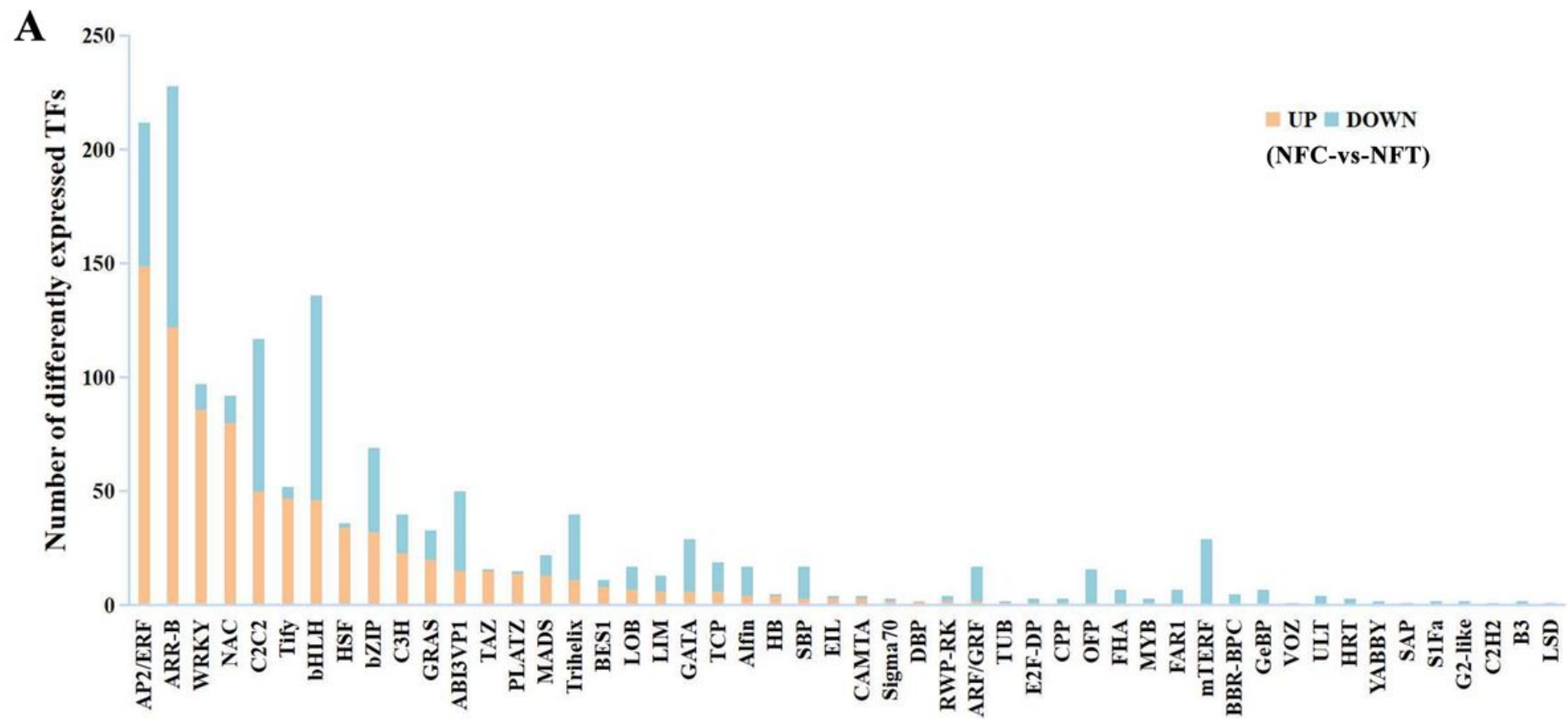

B

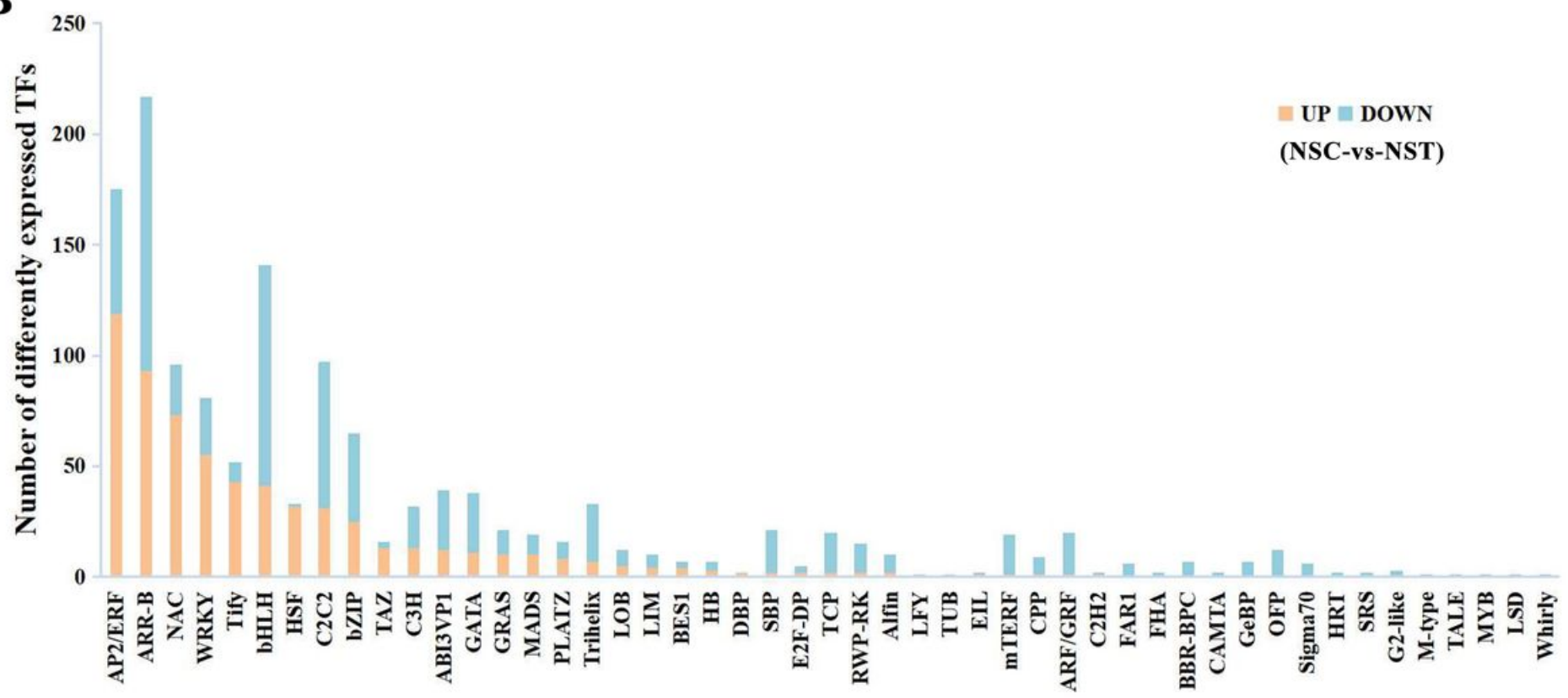

\section{Figure 7}

Classification of differentially expressed TFs in developing leaves of winter rapeseed under freezing treatment. TFs detected in transcriptome of rapeseed cultivars NF (A) and NS (B), respectively. 


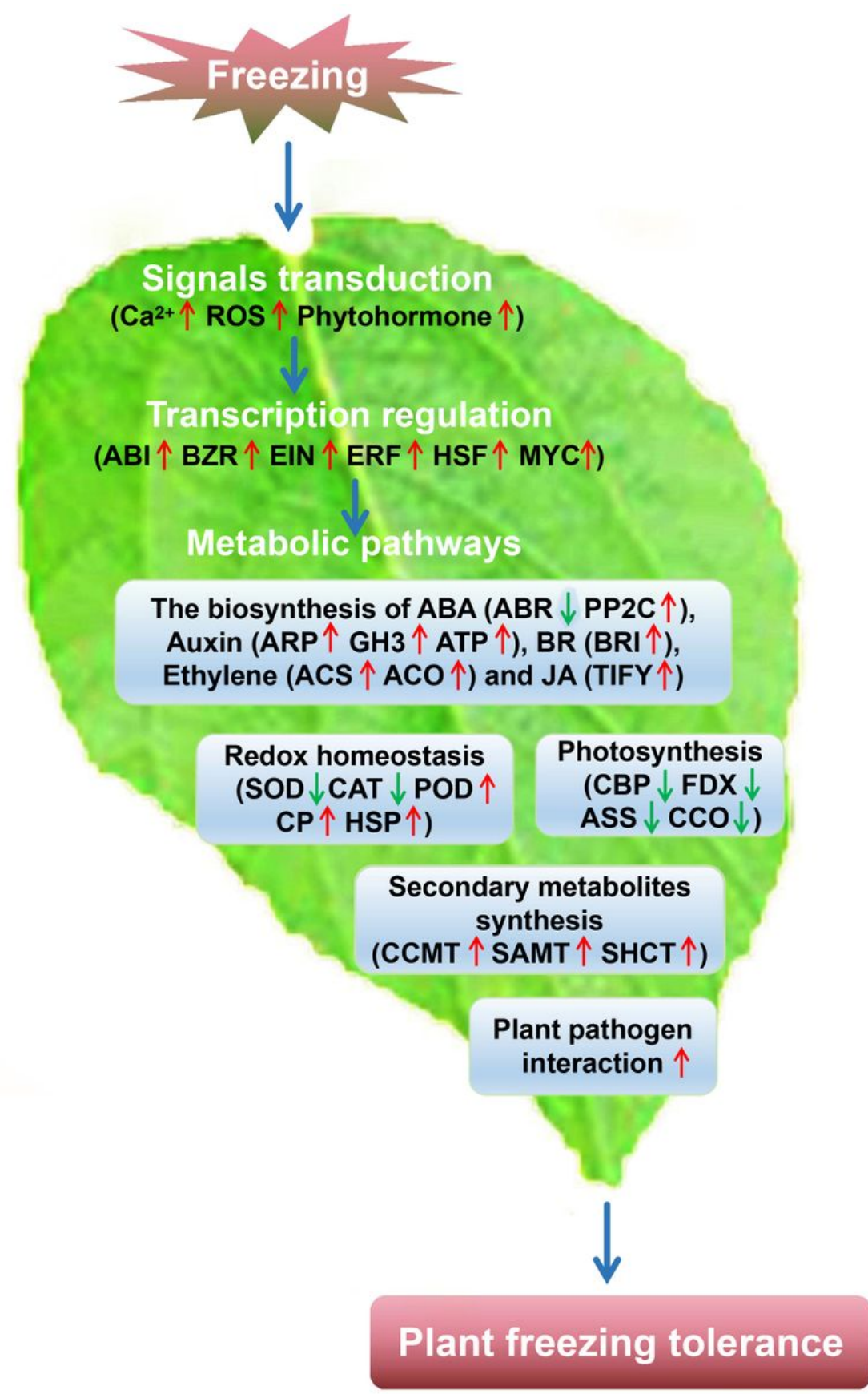

Figure 8

A speculated freezing responsive mechanism in developing leaves of winter rapeseed under freezing treatment. The upregulated freezing responsive genes and proteins are marked by the red " $\uparrow$ " and those down-regulated marked by the green"」”.

\section{Supplementary Files}

This is a list of supplementary files associated with this preprint. Click to download.

- FIGS1.jpg

- FIGS2.jpg

- FIGS3.jpg 
- FIGS4.jpg

- FIGS5.jpg

- Tables1.xlsx

- Tables2.xlsx

- Tables3.xlsx

- TableS4.xlsx

- TableS5.xIsx

- Tables6.xIsx 